\title{
Remote Strain Sensing of CFRP using Microwave Frequency Domain Reflectometry
}

\author{
William C. Wilson, Jason P. Moore, Peter D. Juarez \\ Nondestructive Evaluation Sciences Branch \\ NASA Langley Research Center \\ Hampton, VA, USA \\ William.C.Wilson@nasa.gov
}

\begin{abstract}
NASA's Advanced Composites Project is investigating technologies that increase automated remote inspection of aircraft composite structures. Therefore, microwave Frequency Domain Reflectometry (FDR) is being investigated as a method of enabling rapid remote measurement of strain occurring at the first ply of a composite fiber reinforced polymer (CFRP) structure using Radio Frequency (RF) Electro-Magnetic (EM) radiation. While microwave reflectometry has been used to detect disbonds in CFRP structures, its use in detecting strain has been limited. This work will present data demonstrating the measurement of the reactance changes due to loading conditions that are indicative of strain in a CFRP structure. In addition, the basic EM signature will be presented along with an analysis of temperature and humidity effects.
\end{abstract}

Keywords- Microwave, Frequency Domain Reflectometry, Electromagnetic; CFRP; strain, $R F$

\section{INTRODUCTION}

NASA's Advanced Air Vehicles Program (AAVP) the Advanced Composites Project is investigating technologies that increase aircraft component inspection throughput by $30 \%$ through the development of quantitative and practical inspection methods [1]. Automated remote inspection of composite structures is required to reduce inspections times and yield improved strain data. Microwave Frequency Domain Reflectometry (FDR) is being investigated as a method of enabling rapid remote measurement of strain occurring at the first carbon fiber layer of a composite fiber reinforced polymer (CFRP) structure using Radio Frequency (RF) Electro-Magnetic (EM) radiation.

Microwave energy has been used for nondestructive evaluation in the past [2], and microwave reflectometers have been used to detect disbonds in CFRP structures [3]. Microwaves have also been used to examine the Space Shuttle Columbia's Spray On Foam Insulation (SOFI) and for the inspection of shuttle reentry tiles $[4,5]$. A microwave thickness gauge was developed to measure the thickness of liquid shim materials used to bond aircraft composite skins to wing spars [6]. Microwave non-contact reflection systems (sometimes using two antennas) have even been used to measure breathing and heart rate of patients [7-10]. It should be noted that the previous efforts have used microwave energy to image composite structures, however, it has not been used to measure strain in the structure.
It has been found that strain causes a change in the electrical resistance and impedance of the carbon fibers within CFRP structures when electrodes are wired to the structure [11-13]. Therefore, the impedance (resistance and reactance) changes due to loading should be detectable through changes in the frequency response when interrogated using microwaves. This work will investigate impedance changes due to strain changes in a CFRP panel.

\section{EXPERIMENTAL SETUP}

For this work, a Carbon fiber-reinforced polymer (CFRP) composite panel measuring $406 \mathrm{~mm}$ by $406 \mathrm{~mm}$ by $2.78 \mathrm{~mm}$ was fabricated at NASA Langley Research Center. The composite laminate IM7/8552 is quasi-isotropic and is made up of IM7 fibers and 8552 prepreg material with a 26 ply thick layup of $\left[(0 /+45 /-45 / 90)_{3} 0\right]_{\mathrm{s}}$. Details of the material properties are available in a prior paper by Leckey et al [14]. The test fixture consists of a $90^{\circ}$ angle bracket bolted to an optics breadboard. The top of the panel is bolted to the angle bracket and the bottom of the panel hangs freely. This arrangement allows for weights to be placed at the center of the bottom edge to load the panel (Fig 1.).

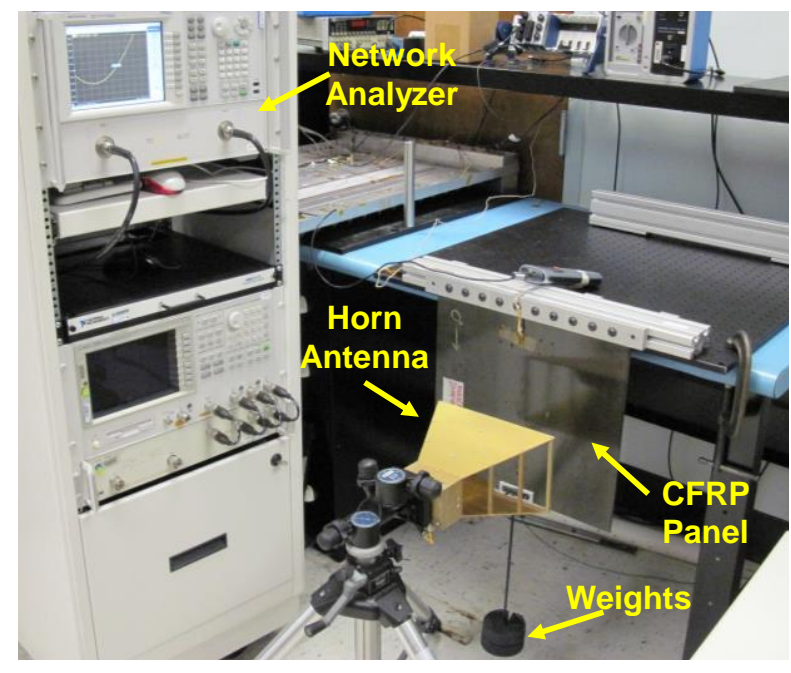

Fig. 1. Experimental setup, CFRP panel, weights and horn antenna. 


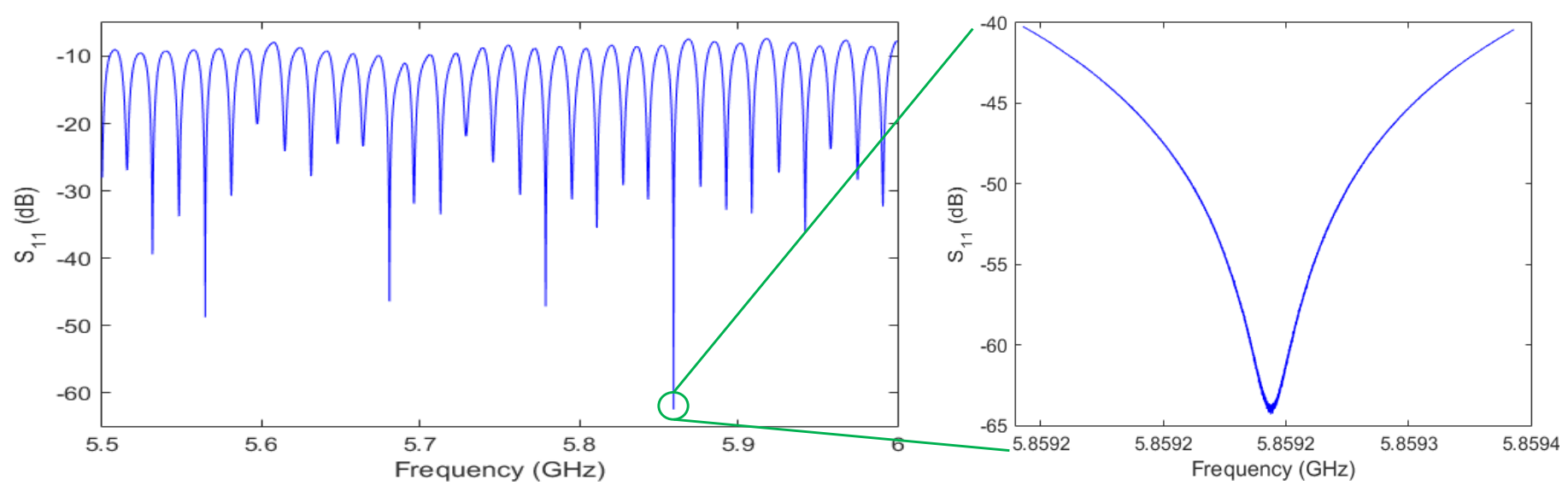

Fig. 2. (a) The EM signature ( $\mathrm{S}_{11}$ response) of the CFRP panel from $5.5 \mathrm{GHz}$ to $6 \mathrm{GHz}$. (b) The $\mathrm{S}_{11}$ response for the $200 \mathrm{kHz}$ bandwidth used for this work.

It has been found that for multi-ply CFRP panels the reflectance drops to $\sim 90 \%$ at $10 \mathrm{GHz}$ and drops further to $\sim 30 \%$ at $60 \mathrm{GHz}$ [15]. To ensure that the reflectivity is above $90 \%$, this work will use frequencies around $5.859 \mathrm{GHz}$. Additionally, the absorption of microwaves creates heat in the composite structure, therefore this work will utilize frequencies that are largely reflective and avoid unnecessary heating of the sample under test. The system is a microwave reflectometer so a single antenna is used for both sending and receiving the RF energy. The network analyzer transmits a chirp signal from 5.85915 $\mathrm{GHz}$ to $5.85935 \mathrm{GHz}$, and subsequently receives the reflected signal and captures the $S_{11}$ reflection parameters. By tracking the changes in the $S_{11}$ data, strain measurements may be achieved.

The composite panel is interrogated using an Agilent N5230C vector network analyzer connected to an A. H. Systems SAS-571, $700 \mathrm{MHz} \sim 18 \mathrm{GHz}$ horn antenna located $120 \mathrm{~mm}$ from the panel. In addition to the antenna, a thermocouple is attached to the panel to measure the temperature, and a hygrometer is located above the panel to measure the humidity. The thermocouple is monitored using a National Instruments cDaq chassis which is connected to a computer via USB. The network analyzer is likewise connected to the computer via USB. The humidity is monitored with a Rotronic Hygro Palm HP21 hygrometer connected to the computer via USB. All data is collected and stored using custom LabVIEW code.

\section{RESULTS}

Before taking data the basic $S_{11}$ response of the CFRP was measured from $5.5 \mathrm{GHz}$ to $6 \mathrm{GHz}$ (Fig. 2 (a)). A frequencychirped signal is transmitted to the panel through the horn antenna and the complex $S_{11}$ response is captured through the same antenna. The CFRP response has many peaks and troughs. The trough with the lowest amplitude and greatest amplitude change was chosen for this work, Fig. 2 (b). As a result, the frequency band of $5.85915 \mathrm{GHz}$ to $5.85935 \mathrm{GHz}$ was selected. These frequencies were used for interrogating strain in the CFRP panel.
For a thorough characterization of the $\mathrm{S}_{11}$ response, the data is first converted into the complex impedance (Z) using:

$$
\mathrm{Z}=\mathrm{Z}_{0}\left(1+\mathrm{S}_{11}\right) /\left(1-\mathrm{S}_{11}\right)
$$

where $Z_{0}$ is the load impedance or in this case the instrument impedance $(50 \Omega)$, and $S_{11}$ is the complex reflection coefficient from the network analyzer.

The $S_{11}$-derived impedance, temperature, and humidity were sampled 20 times at mass loads $0 \mathrm{~kg}$ to $10 \mathrm{~kg}$ in $2 \mathrm{~kg}$ increments and then again at $0 \mathrm{~kg}$; this process was repeated three times. The magnitude of the impedance for the three load cycles is given in Fig. 3.

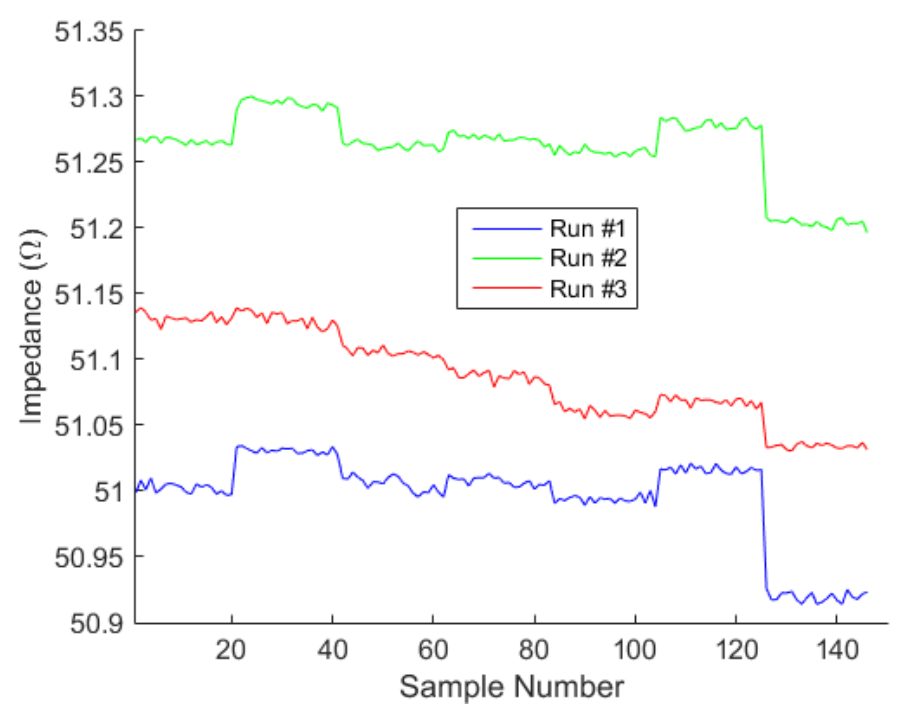

Fig. 3. The magnitude of the impedance data for the three load cycles. 
While the impedance magnitude does not show an obvious correlation to strain changes, further investigation in future work may lead to using magnitude measurements to further characterize CFRP layups. Because the impedance is complex, it can be defined as:

$$
\mathrm{Z}=\mathrm{R}+\mathrm{Xj}
$$

where $\mathrm{R}$ is the resistance or the real component of the complex impedance data, and $\mathrm{X}$ is the reactance or imaginary component of the complex impedance data which corresponds to either capacitance or inductance. Fig. 3 shows the magnitude, the resistance, and the reactance of the impedance data from the first loading cycle (at each loading step the mass was kept constant for 20 samples).

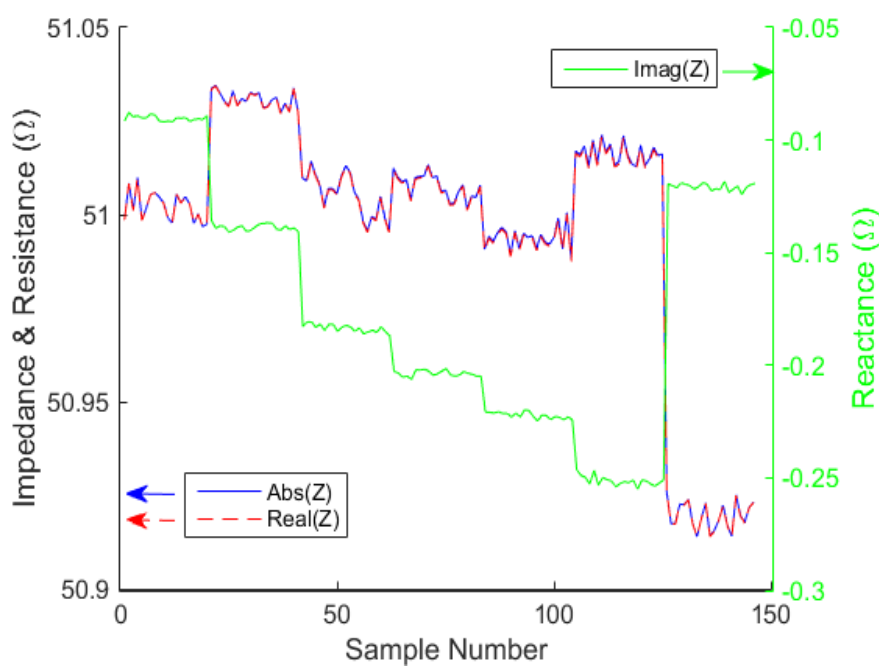

Fig. 3. The Impedance, Resistance and Reactance response of the CFRP panel when loaded from $0 \mathrm{~kg}$ to $10 \mathrm{~kg}$ and back to $0 \mathrm{~kg}$ in $2 \mathrm{~kg}$ increments (load cycle \#1).

The magnitude of the impedance is dominated by the resistance values, while the much smaller reactance appears to have a greater, more consistent, dependence on strain changes induced by panel loading. Although the reactance changes with strain, the changes are not linear. The cause is most likely environmental effects such as temperature and humidity changes during the measurement process that effect the resistance more than either the inductance or capacitance. Three separate cycles yield reactance data with consistent steps due to increasing load however the overall amplitude of the reactance data varies widely between load cycles (Fig. 4.)

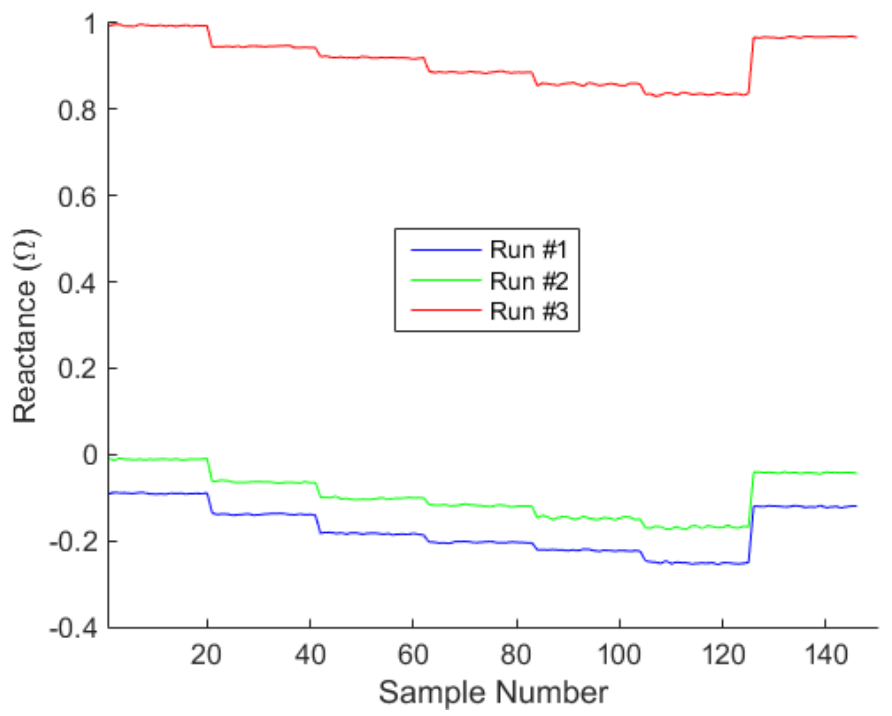

Fig. 4. Reactance data for the three load cycles.

The reactance data shows an inverse relationship to the strain, as the reactance decreases as the strain increases. This effect was expected and is due to the negative piezoresistivity found in carbon fibers [16]. It is suspected that the differing offsets in the reactance data between load cycles is due to environmental effects of temperature and humidity. To investigate the environmental effects both temperature and humidity were recorded at each $S_{11}$ sample point. The 20 temperature and humidity measurements recorded at each load step have been averaged together for each point in Fig.5 and Fig.6, respectively.

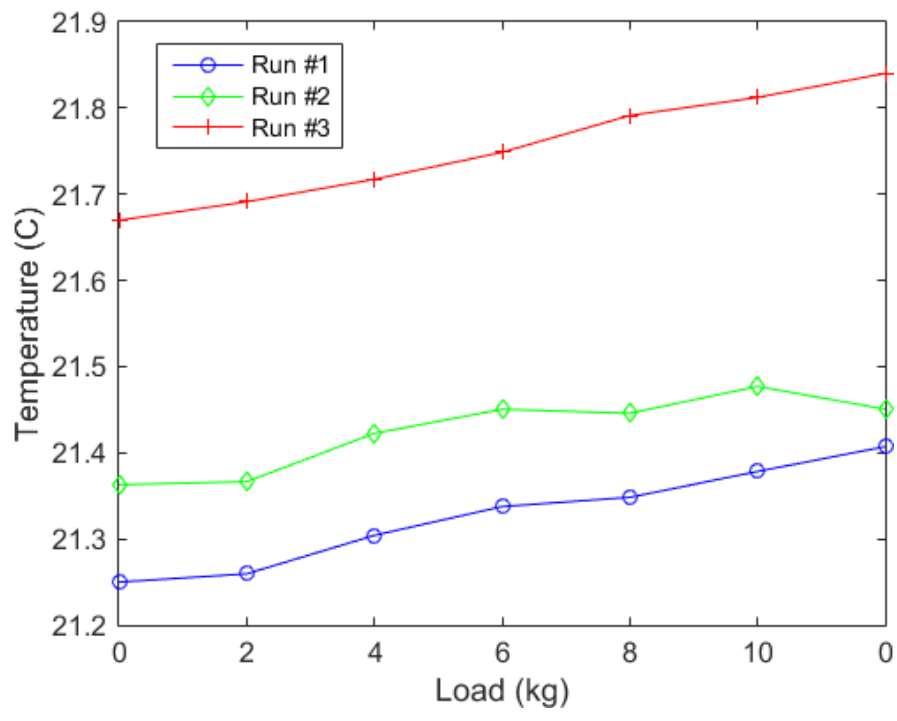

Fig. 5. Thermocouple data for the three load cycles.

The temperature data shows differing offset between load cycles but drifts upward during each load cycle. 


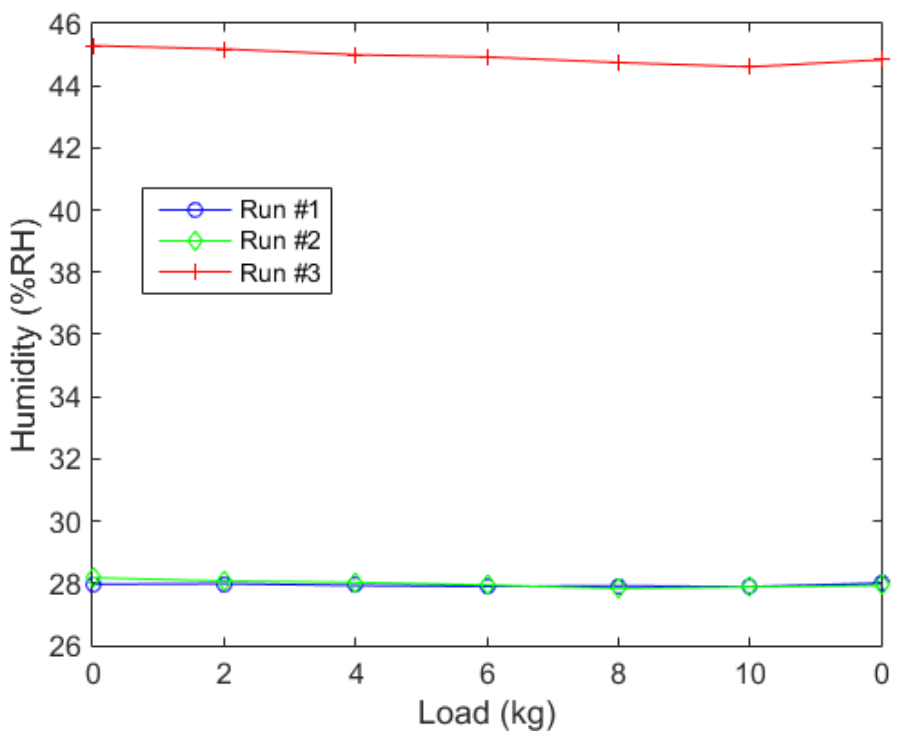

Fig. 6. Humidity data for the three load cycles.

The three load cycles were conducted on different days; apparently the third load cycle was performed on a particularly humid day. Both humidity and temperature are known to change the impedance of CFRP, so both will need to be measured and used for compensation of any strain measurements [17].

For comparison the reactance data was averaged across 20 samples at each load condition (Fig. 7).

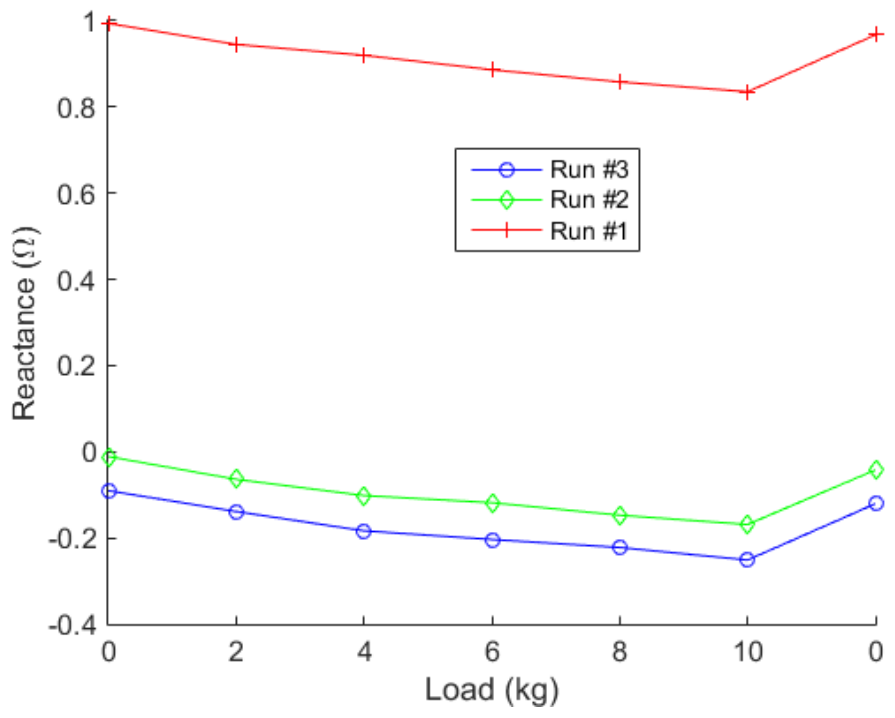

Fig. 7. Reactance data for the three load cycles.

The step-changes in reactance within each load cycle are a result of strain due to loading while the varying offsets between cycles is due to differing temperature and humidity conditions. The offset in cycle 3 is clearly influenced by the higher humidity measured during the cycle. To compensate the reactance measurements to match strain, both the temperature and humidity measurements are used. A linear fit for reactance vs. load is found for each load cycle, giving 0.0016 ohms per Newton force on average. To compensate the measurements to the linear fits and to normalize the measurements of each cycle to zero reactance at zero load, both temperature and humidity are used in a multivariable regression algorithm with the difference between the linear fits and the data used as the target. The compensation equation is given by:

$$
X=p_{1}+p_{2} H+p_{3} T+p_{4} H^{2}+p_{5} H T+p_{6} T^{2}+p_{7} H^{3}
$$

where $X$ is the compensated reactance, $H$ is the humidity, $T$ is the temperature, and $p_{n}$ are the coefficients. The seven coefficients for the three load cycles are averaged together to give the set of coefficients that are used for this work. See Table 1 for list of all of the coefficients.

TABLE I. FITTING COEFFICIENTS

\begin{tabular}{|l|c|c|c|c|}
\hline & Cycle \#1 & Cycle \#2 & Cycle \#3 & Average \\
\hline $\mathbf{p}_{\mathbf{1}}$ & $-8.6736 \mathrm{e}-19$ & $-1.7347 \mathrm{e}-18$ & $-8.6736 \mathrm{e}-19$ & $-1.1565 \mathrm{e}-18$ \\
\hline $\mathbf{p}_{2}$ & $2.4216 \mathrm{e}+00$ & $1.5633 \mathrm{e}+01$ & $6.2137 \mathrm{e}-01$ & $5.6253 \mathrm{e}+00$ \\
\hline $\mathbf{p}_{3}$ & $1.3666 \mathrm{e}+00$ & $3.0808 \mathrm{e}+01$ & $5.7068 \mathrm{e}+00$ & $1.4074 \mathrm{e}+01$ \\
\hline $\mathbf{p}_{4}$ & $5.1856 \mathrm{e}+01$ & $1.7572 \mathrm{e}+02$ & $1.2391 \mathrm{e}+00$ & $5.9400 \mathrm{e}+01$ \\
\hline $\mathbf{p}_{5}$ & $-6.2728 \mathrm{e}+01$ & $7.6049 \mathrm{e}+02$ & $-2.1621 \mathrm{e}+00$ & $2.5206 \mathrm{e}+02$ \\
\hline $\mathbf{p}_{6}$ & $-2.6194 \mathrm{e}+00$ & $1.0611 \mathrm{e}+03$ & $-3.8119 \mathrm{e}+01$ & $3.2829 \mathrm{e}+02$ \\
\hline $\mathbf{p}_{7}$ & $1.0718 \mathrm{e}+03$ & $8.0798 \mathrm{e}+01$ & $1.0374 \mathrm{e}-01$ & $2.7002 \mathrm{e}+01$ \\
\hline
\end{tabular}

Applying the averaged coefficients to the data yields the compensated reactance values for each load cycle (Fig. 8).

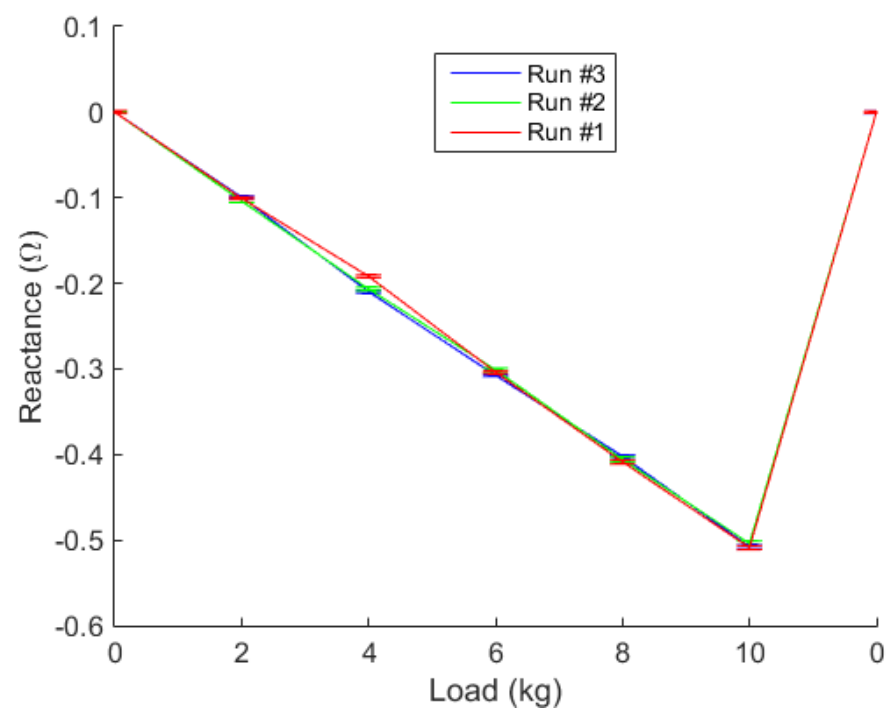

Fig. 8. Compensated reactance values for each of the three load cycles.

The compensated data is fairly linear and the steps are more consistent after processing. As stated before, the reactance has an inverse relationship with the loading (strain). The error bars in Fig. 8, indicate one standard deviation. The data has 0.3024 ohms per $\mu \varepsilon$, or 0.0016 ohms per Newton of force. 
To demonstrate that RF can be used to measure strain, the strain due to mass loading is modeled using the standard equation for a rectangular cross section structure which is given by:

$$
\varepsilon=\frac{\delta}{L}=\frac{F}{E A}
$$

where $\varepsilon$ is the strain, $\delta$ is the elongation due to the applied force $\mathrm{F}, \mathrm{E}$ is young's modulus ( $\mathrm{E}=171.4 \mathrm{GPa}$ for the panel) and A is the area. The load values were converted to Newtons of force for the model. Using the model, the maximum load of $10 \mathrm{~kg}$ yields a strain of $0.507 \mu \varepsilon$ for the panel.

Shown in Fig. 9 is the data-derived strain and modelpredicted strain vs. panel load. The compensated reactance measurements and the model-predicted strain at each load point gives a $3.31 \mu \varepsilon / \Omega$ conversion factor.

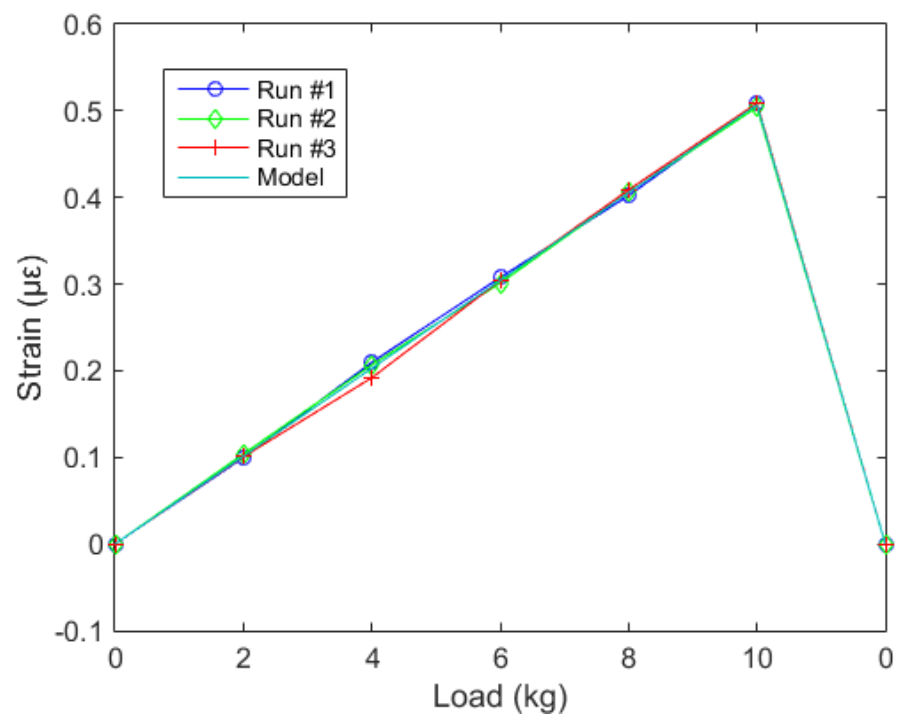

Fig. 9. Experimental strain and model strain data for the three load cycles.

The steps are monotonic and roughly linear $(0.0507 \mu \varepsilon / \mathrm{kg})$. Note that all three runs return to zero. This initial compensated data-derived strain matches fairly closely to the model-predicted values. However, the differences may be reduced through optimization of the test setup or an altered compensation model.

The difference between the model-predicted strain and the compensated reactance-based strain for the three load cycles is shown in Fig. 10. The compensation method functions adequately for this initial investigation, as the error between the model and the compensated reactance values is between $6.944 \mathrm{e}-03 \mu \varepsilon$ and $1.0900 \mathrm{e}-02 \mu \varepsilon(\sim 5.5 \%$ error max).

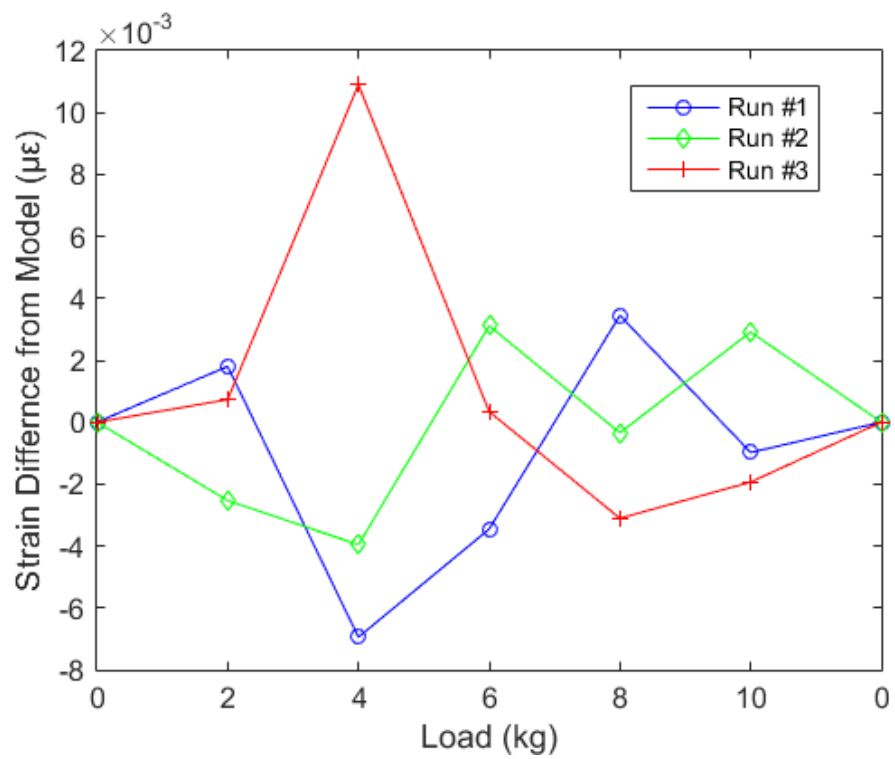

Fig. 10. Strain difference data between measured and model for the three load cycles.

To demonstrate the effectiveness of the measurement process and compensation method, the reactance, temperature, and humidity were recorded for three additional load cycles of the panel. Using the previously determined compensation coefficients and the $3.31 \mu \varepsilon / \Omega$ conversion factor gives the panel strain measurements shown in Fig. 11.

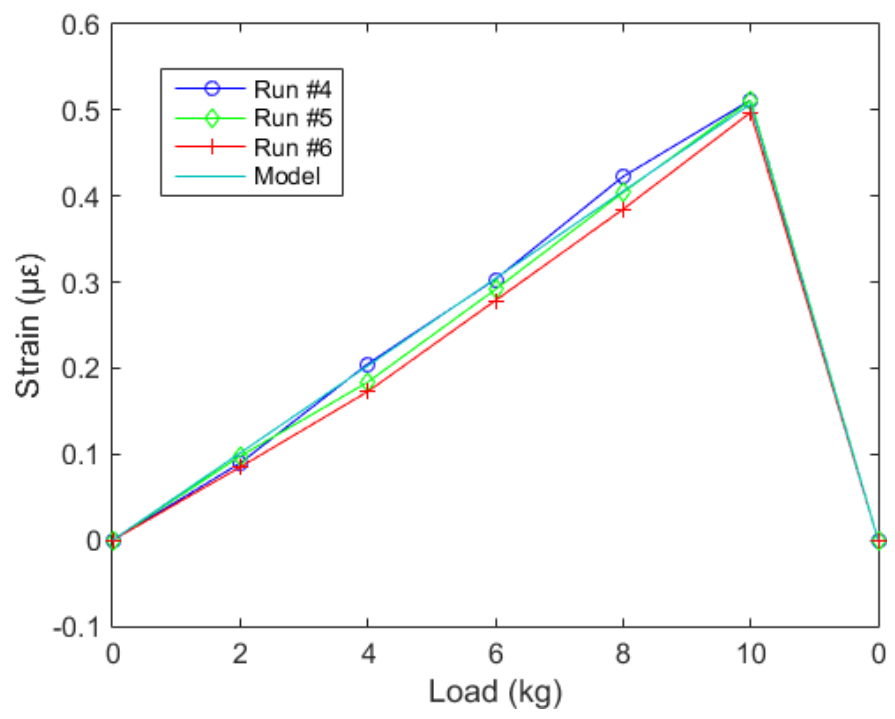

Fig. 11. Strain and model strain data for three additional load cycles.

Included in Fig. 11 are the model-predicted panel strain values at each load. While the compensated measurements do not match the model perfectly they are reasonably close. The difference between the compensated measurements and the model prediction for the three additional load cycles is given in Fig. 12. 


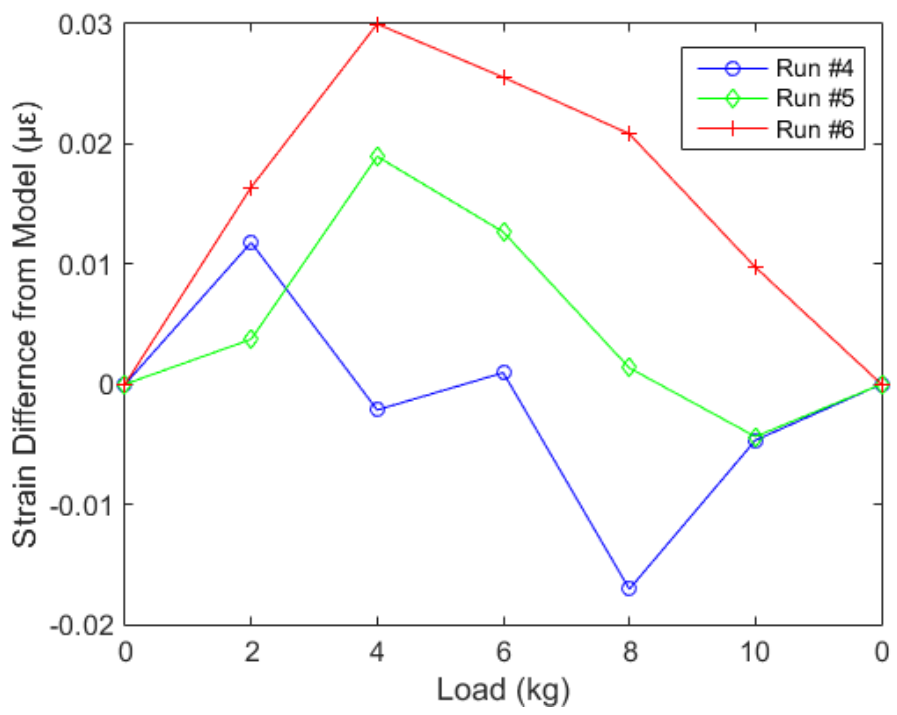

Fig. 12. Difference data between strain and model for the three additional load cycles.

The microwave-based measurement agrees well with the model-predicted strain, with error from the model between $-0.01706 \mu \varepsilon$ to $0.02998 \mu \varepsilon$, for the last three load cycles.

The difference data reveals that although the RF data in general follows the strain, it has large variations between the load cycles. Further characterization of the temperature and humidity effects may help to reduce the variations between measurements. Note that the maximum strain for this work is only $0.507 \mu \varepsilon$; larger loading of the panel, via a load-frame for example, would most likely reduce the percentage error. However the device does demonstrate sub-micro strain measurement capabilities. Further optimization of the test setup may also increase the signal to noise ratio.

\section{DISCUSION}

A microwave reflectometer has been used to demonstrate remote sensing of reactance that is indicative of strain within a CFRP panel. It was determined that the reactance vales can be used to measure strain with a $3.31 \mu \varepsilon / \Omega$ conversion factor, which leads to a resolution of $0.0507 \mu \varepsilon / \mathrm{kg}$ for the chosen CFRP panel. This non-contact method of measuring strain after fabrication supports efforts to automate aircraft composite inspections.

This work is preliminary, therefore, optimization of the test setup, or further post processing, and further characterization of the temperature and humidity effects may help to reduce the variations between runs and reduce the difference between the measurements and the strain model.

\section{REFERENCES}

[1] Techport, "Advanced Composites Project, Advanced Air Vehicles Program, Aeronautics Research Mission Directorate (ARMD)," NASA Langley Research Center, Project Status Report, URL: https://techport.nasa.gov/view/13280, 2016, p. 9.

[2] S. Kharkovsky and R. Zoughi, "Microwave and Millimeter Wave Nondestructive Testing and Evaluation-Overview and Recent Advances," Instrumentation \& Measurement Magazine, IEEE, vol. 10, no. 2, pp. 26-38, 2007.

[3] S. Kharkovsky, A. Ryley, et al., "Dual-Polarized Near-Field Microwave Reflectometer for Noninvasive Inspection of Carbon Fiber Reinforced Polymer-Strengthened Structures," IEEE Trans. on Instrumentation and Measurement, vol. 57, no. 1, pp. 168-175, Jan. 2008.

[4] J. Case, F. Hepburn, et al., "Inspection of Spray on Foam Insulation (SOFI) using Microwave and Millimeter Wave Synthetic aperture Focusing and Holography," in Proc. Instrumentation and Measurement Technology Conference, 2006. IMTC 2006. Proceedings of the IEEE, Sorento, Italy, April 24-27, 2006, pp. 2148-2153.

[5] R. Zoughi, S. Kharkovsky, et al., "Microwave and Millimeter Wave Testing for the Inspection of the Space Shuttle Spray on Foam Insulations (SOFI) and the Acreage Heat Tiles," in Proc. AIP Conf., Quantitative Nondestructive Evaluation, Vol. 820, Brunswick, ME, July 31-5, 2005, pp. 439-446.

[6] D. Palmer, S. Buckner, et al., "Evaluation of Microwave Methods for Thickness Measurements of Liquid Shim Material," in Review of Progress in Quantitative Nondestructive Evaluation, Springer, vol. 12, 1993, pp. 1955-1962.

[7] D. Dei, G. Grazzini, et al., "Non-Contact Detection of Breathing using a Microwave Sensor," Sensors, vol. 9, no. 4, pp. 2574-2585, 2009.

[8] J. Lin and C. Li, "Wireless Non-Contact Detection of Heartbeat and Respiration Using Low-Power Microwave Radar Sensor," in Proc. AsiaPacific Microwave Conference, APMC'07. , Dec. 11-14, 2007, pp. 1-4.

[9] A. D. Droitcour, "Non-Contact Measurement of Heart and Respiration Rates with a Single-Chip Microwave Doppler Radar," PhD, Department of Electrical Engineering, Stanford University, 2006, p. 470.

[10] G. Lu, F. Yang, et al., "Contact-Free Measurement of Heart Rate Variability via a Microwave Sensor," Sensors, vol. 9, no. 12, pp. 95729581, Nov. 302009.

[11] A. Todoroki, M. Ueda, et al., "Strain and Damage Monitoring of CFRP Laminates by Means of Electrical Resistance Measurement," Journal of Solid Mechanics and Materials Eng., vol. 1, no. 8, pp. 947-974, 2007.

[12] J. Abry, S. Bochard, et al., "In Situ Detection of Damage in CFRP Laminates by Electrical Resistance Measurements," Composites Science and Technology, vol. 59, no. 6, pp. 925-935, 1999.

[13] T. A. Ezquerra, M. T. Connor, et al., "Alternating-Current Electrical Properties of Graphite, Carbon-Black and Carbon-Fiber Polymeric Composites," Composites science and technology, vol. 61, no. 6, pp. 903-909, May 2001.

[14] C. A. Leckey, M. D. Rogge, et al., "Guided Waves in Anisotropic and Quasi-Isotropic Aerospace Composites: Three-Dimensional Simulation and Experiment," Ultrasonics, vol. 54, no. 1, pp. 385-394, 2014.

[15] J. H. Kaiser, "Reflection and Absorption of Microwaves (2-100 GHz) by Carbon-Fiber-Reinforced Composite Surfaces," Research in Nondestructive Evaluation, vol. 5, no. 4, pp. 275-283, 1994.

[16] S. Wang and D. Chung, "Negative Piezoresistivity in Continuous Carbon Fiber Epoxy-Matrix Composite," Journal of materials science, vol. 42, no. 13, pp. 4987-4995, 2007.

[17] S. Wang, D. P. Kowalik, et al., "Effects of the Temperature, Humidity, and Stress on the Interlaminar Interface of Carbon Fiber Polymer-Matrix Composites, Studied by Contact Electrical Resistivity Measurement," The Journal of Adhesion, vol. 78, no. 2, pp. 189-200, 2002. 